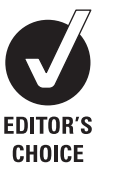

\title{
Substance $P$ accelerates hypercellularity and angiogenesis in tendon tissue and enhances paratendinitis in response to Achilles tendon overuse in a tendinopathy model
}

\author{
Gustav Andersson, ${ }^{1}$ Ludvig J Backman, ${ }^{1}$ Alexander Scott, ${ }^{2,3}$ Ronny Lorentzon, ${ }^{4}$ \\ Sture Forsgren, ${ }^{1}$ Patrik Danielson ${ }^{1}$
}

1Department of Integrative Medical Biology, Anatomy Section, Umeå University, Umeå, Sweden

${ }^{2}$ Department of Physical Therapy, University of British Columbia, Vancouver, British Columbia, Canada

${ }^{3}$ Centre for Hip Health and

Mobility, Vancouver Coastal Health and Research Institute, Vancouver, British Columbia, Canada

${ }^{4}$ Department of Surgical and Perioperative Sciences, Sports Medicine Unit, Umeå University, Umeå, Sweden

\section{Correspondence to}

Dr Patrik Danielson,

Department of Integrative Medical Biology, Anatomy Section, Umeå University, SE-901 87 Umeå, Sweden; patrik.danielson@anatomy. umu.se

Accepted 12 March 2011

Published Online First

2 May 2011

\section{ABSTRACT}

Background Tenocytes produce substance P (SP), and its receptor (neurokinin-1 receptor (NK-1R)) is expressed throughout the tendon tissue, especially in patients with tendinopathy and tissue changes (tendinosis) including hypercellularity and vascular proliferation. Considering the known effects of SP, one might ask whether SP contributes to these changes.

Objectives To test whether development of tendinosislike changes (hypercellularity and angiogenesis) is accelerated during a 1-week course of exercise with local administration of SP in an established Achilles tendinopathy model.

Methods Rabbits were subjected to a protocol of Achilles tendon overuse for 1 week, in conjunction with $\mathrm{SP}$ injections in the paratenon. Exercised control animals received $\mathrm{NaCl}$ injections or no injections, and unexercised, uninjected controls were also used. Tenocyte number and vascular density, as well as paratendinous inflammation, were evaluated. Immunohistochemistry and in situ hybridisation to detect NK-1R were conducted.

Results There was a significant increase in tenocyte number in the SP-injected and NaCl-injected groups compared with both unexercised and exercised, uninjected controls. Tendon blood vessels increased in number in the SP-injected group compared with unexercised controls, a finding not seen in $\mathrm{NaCl}$ injected controls or in uninjected, exercised animals. Paratendinous inflammation was more pronounced in the $\mathrm{SP}$-injected group than in the $\mathrm{NaCl}$ controls. NK-1R was detected in blood vessel walls, nerves, inflammatory cells and tenocytes.

Conclusions SP accelerated the development of tendinosis-like changes in the rabbit Achilles tendon, which supports theories of a potential role of $\mathrm{SP}$ in tendinosis development; a fact of clinical interest since $\mathrm{SP}$ effects can be effectively blocked. The angiogenic response to SP injections seems related to paratendinitis.

\section{INTRODUCTION}

The potential role of substance P (SP) in tendons has gained increased interest in recent years (see ref ${ }^{1}$ for a review). Most studies performed on SP and tendons to date have focused on healing processes after tendon rupture (eg, see refs ${ }^{2-5}$ ) but there are also potential roles of SP in tendinosis, that is, chronic tendon pain (tendinopathy) with degenerative-like tissue changes, which warrant further investigation. ${ }^{4}$
In addition to its established role in peripheral pain, SP has known proinflammatory effects and effects on vasodilation and vascular permeabilisation, ${ }^{67}$ as well as reparative effects including angiogenesis ${ }^{8}$ and cell proliferation, the latter also in tendon tissue. ${ }^{34}$ We recently demonstrated in human Achilles tendons that tenocytes produce SP $\mathrm{mRNA}^{9}$ and that the preferred receptor for SP, the neurokinin-1 receptor (NK-1R), is widely distributed in blood vessel walls, nerve fascicles and tenocytes of the tendon tissue. ${ }^{9} 10$ Evidence of SP production and NK-1R expression were particularly prominent in tendons displaying characteristic features of tendinosis (hypercellularity and neovascularisation). There are also findings suggesting an increase in SP-immunoreactive nerve fibres and an increase in mast cell numbers in tendinosis. ${ }^{11} 12$ The potential for effects of SP in Achilles tendons includes modulation of angiogenesis and/or vascular regulation, tenocyte and mast cell function and pain signalling.

Healing of ruptured Achilles tendons is associated with an ingrowth of SP-positive nerve fibres. ${ }^{13}$ This finding has led to the hypothesis that exogenously administered SP could actually facilitate tendon healing. Indeed, paratendinous injection of SP enhanced cell proliferation, angiogenesis and collagen reorganisation during rat Achilles tendon healing. ${ }^{34}$ In light of this, it is interesting to note that tendinosis is often characterised as a process of failed tendon healing due to an imbalance between reparative capacity and ongoing repetitive microtrauma. ${ }^{14}{ }^{15}$ In fact, some cardinal features of tendinosis are commonly observed in tendon healing after rupture, particularly tenocyte proliferation and neovascularisation. ${ }^{16}$ It could therefore be hypothesised that SP has a role in tendinosis development by exacerbation of repeated inflammation-repair responses.

To test this hypothesis, we used a model of Achilles tendon overuse, ${ }^{17}$ based on the studies by Backman and collaborators, ${ }^{18} 19$ in which we recently demonstrated that tendinosis-like tissue changes (tenocyte hypercellularity and vascular proliferation) occur after 3 weeks of exercise, but not after a single week of exercise. ${ }^{17}$ Specifically, we aimed to determine whether repetitive injections of SP to the paratendinous tissue would accelerate the development of tendinosis-like changes in the rabbit Achilles tendon. 


\section{MATERIAL AND METHODS}

Animals

Twenty-four mature female New Zealand white rabbits were randomly divided into four groups of six; one control group was not subjected to the overuse protocol ('untrained controls'), two groups were subjected to this protocol for 1 week and were given repetitive injections of SP ('SP-injected group') or saline ('NaCl-controls'), respectively, and one group was subjected to the protocol for 1 week without injections ('1-week controls'). The untrained controls and the 1-week controls were also analysed in a previous study. ${ }^{17}$

\section{Experimental design}

An animal model shown to induce tendinosis-like changes ${ }^{17} 18$ was used. The apparatus produced passive dorsiflexion and plantar flexion of the right ankle in combination with a controlled, concentric, muscle contraction through electrical stimulation. The exercise regimen was performed for $2 \mathrm{~h}$, every second day, for 1 week. The left leg was not attached to the apparatus. For details, see ref ${ }^{17}$.

Anaesthesia was administered by intramuscular injection of fentanylfluanison $(0.2-0.3 \mathrm{ml} / \mathrm{kg})$ and diazepam $(5 \mathrm{mg} / \mathrm{ml}$, $0.2 \mathrm{ml} / \mathrm{kg}$ ). An additional $0.1 \mathrm{ml} / \mathrm{kg}$ of fentanylfluanison was given every 30-45 min. Subcutaneous buprenorphine (0.01-0.05 $\mathrm{mg} / \mathrm{kg}$ ) was given after each training session. Experiments were approved by the local ethics committee for animal research.

\section{Injection treatments}

In addition to the above described training regimen, injections of SP $\left(10^{-8} \mu \mathrm{mol}\right.$, Sigma, Saint Louis, Missouri, USA: code $\mathrm{S} 6883$; in $1 \mathrm{ml}$ sterile water) or, as control, $0.9 \% \mathrm{NaCl}(1 \mathrm{ml})$ were given immediately following each exercise session in the connective tissue ventral to the Achilles tendon. The group injected with SP received bilateral injections, as we previously noted that the overuse protocol lead to bilateral changes. ${ }^{17}$ In the $\mathrm{NaCl}$ control group, one rabbit was lost due to adverse effects of anaesthesia.

\section{Sampling, fixation and sectioning}

One day after the last exercise, the rabbits were killed with an overdose of pentobarbital. Achilles tendon was collected and biopsies of approximately $5 \times 5 \mathrm{~mm}$ were gathered from the distal tendon (adjacent to the calcaneal insertion), the midportion of the tendon and the muscle-tendon junction (proximal part of the tendon).

Samples from all animals were either immediately frozen in propane chilled with liquid nitrogen or fixed in $4 \%$ formaldehyde and washed in Tyrode's solution, before freezing. All biopsies were mounted on cardboard in transverse orientation to the longitudinal axis of the tendons, in optimal cutting temperature compound (TissueTek; Miles Laboratories, Naperville, Illinois, USA), and stored at $-80^{\circ} \mathrm{C}$ until sectioning.

\section{Histological evaluation: tenocytes and inflammation}

$\mathrm{H} \& \mathrm{E}$ staining for morphological evaluation was performed on sections from all samples. Four of the researchers (GA, LB, $\mathrm{SF}, \mathrm{PD}$ ) evaluated the general morphology, including tenocyte morphology and the pattern of collagen organisation, while two (GA, LB) made detailed quantifications of tenocyte numbers in the tendon tissue proper independently, according to the system used in ref ${ }^{17}$. Furthermore, one researcher (GA) graded the degree of inflammation in the paratendinous tissue.
The latter grading was done with a score of $0-3,0$ being 'no visible inflammatory cells' and 3 being 'marked inflammatory infiltration of the tissue'. This grading was performed twice and the mean was calculated for statistical analysis.

Data from the counting of tenocytes concerning tendons from both sides of the untrained controls and the 1-week controls were re-used from a previous study. ${ }^{17}$ In accordance with this previous collection of tendon tissue, tissue from the nonexercised (left) leg of the SP-injected animals was collected for comparison with the tissue from the exercised side.

\section{Immunohistochemistry: receptor patterns and vascular evaluation}

Sections of $7 \mu \mathrm{m}$ thickness were stained with the primary antibodies (Table 1) according to established protocols. ${ }^{9} 17$ Detection was done using a tetramethylrhodamine isothiocyanate-conjugated rabbit antimouse immunoglobulin G (Dako, Denmark), 1:40 (for CD31), or Alexa Fluor 488 (Invitrogen, California, USA), a green dye conjugated to a donkey antigoat antibody, 1:300 (for NK-1R).

Vascularity was graded based on a Bonar scale ${ }^{20}$ that has been modified, ${ }^{17}$ in which each tendon gets a final, combined grade of 0 (normal) to 9 (highly vascular). Analogous to our previous analysis of vascular proliferation in this model, ${ }^{17}$ the mean grade of both tendons for each rabbit was used in these comparisons, since there was no significant difference (Wilcoxon signed rank test) between the two tendons in any of the groups. This was possible for all groups except the $\mathrm{NaCl}$ controls for which the unexercised tendon was not collected.

One observer (GA) graded the vascularity of the slides in a blinded fashion. The previous study has shown the test-retest reliability for this researcher to be good. ${ }^{17}$

Control stainings were performed via a phosphate-buffered saline control and preabsorption of the NK-1R antibody via a blocking peptide from the same manufacturer (sc-5220P, Santa Cruz, California, USA) using a dilution of $50 \mu \mathrm{g} / \mathrm{ml}$.

\section{In situ hybridisation}

Digoxigenin(DIG)-hyperlabelled oligonucleotide probes(ssDNA) were used to detect NK-1R (also known as TACR1) mRNA (GD1001-DS custom designed; GeneDetect, New Zealand) on $10 \mu \mathrm{m}$ sections of unfixed tissue. As the rabbit TACR1 sequence has not yet been cloned, a 'triple probe cocktail' was prepared. This contained three antisense probes directed towards both rat and human TACR1 mRNA and was considered to have a very high probability of detecting the rabbit TACR1 mRNA. The probe cocktail for TACR1 mRNA was used at $50 \mathrm{ng}$ in 15 $\mu \mathrm{l}$ of hybridisation solution. For antisense probe sequences, see Table 2. A 'triple probe cocktail' of the corresponding sense DIGhyperlabelled ssDNA probes was used as negative control. As positive controls, a poly(dT) probe (GD4000-OP; GeneDetect, New Zealand) and a $\beta$-actin antisense probe (GD5000-OP, Gene Detect, New Zealand) were used.

The in situ hybridisation (ISH) procedure has been reported by our laboratory in previous studies, ${ }^{9}$ as well as by other groups using similar techniques upon which our protocol is based. ${ }^{21}$ In this protocol, an alkaline phosphatase (AP)-labelled

Table 1 Details of the primary antibodies used

\begin{tabular}{lllll}
\hline Antigen & Code & Source & Raised in & Dilution \\
\hline CD31 & M0823 & Dako, Glostrup, Denmark & Mouse (monoclonal) & $1: 100$ \\
NK-1R & sc-5220 & Santa Cruz, California, USA & Goat (polyclonal) & $1: 50$ \\
\hline
\end{tabular}


Table 2 Sequences of the digoxigenin-hyperlabelled ssDNA antisense probes in the 'triple probe cocktail' custom designed to detect rabbit TACR1 mRNA (GeneDetect, New Zealand)

\section{Probe sequences}

Probe \# 1: 5'-GGCTGCACGAACTGGTTAGACTCAGAGGTGTTGGTGGAGATGTTGGGG-3' Probe \#2: 5'-TGGAGCTTTCTGTCATGGTCTTGGAGTTGCTGCGAGAGGAGCCGTTGG-3' Probe \#3: 5'-TGACCACCTTGCGCTTGGCAGAGACTTGCTCGTGGTAGCGGTCAGAGG-3'

anti-DIG antibody (Roche, Germany, 11093274 910) is used for detection.

Sections were evaluated by three of the scientists (GA, PD, SF).

\section{Statistics}

The Wilcoxon signed rank test was performed to compare tendons of the same rabbit. The Kruskall-Wallis test was used to test for differences between the groups, followed by pairwise comparisons using the Mann-Whitney $U$ test. The attained $p$ values were multiplied by the number of pairwise tests (Bonferroni correction). These multiplied values are the ones presented in the Results section.

Computer software (PASWStatistics18.0 for Macintosh SPSS Inc, Chicago, Illinois, USA) was used for all statistical calculations, with significance predetermined at $\mathrm{p}<0.05$.

\section{RESULTS}

\section{General morphology}

The tendon tissue proper from the animals of the injected groups was to varying degrees found to display tendinosis-like tissue morphology, ie, occasional tenocytes of abnormal shape, irregular collagen and separation of collagen bundles, similar to that previously noticed for animals that had exercised for 3 weeks or more in the protocol. ${ }^{17}$ This was only rarely noted for the 1-week controls, and not at all for the untrained controls. No evident differences between limbs were noted. However, interindividual variations existed within all groups and the influence on tissue morphology by postharvesting treatment of biopsies (fixation, sectioning etc) made it difficult to draw objective conclusions concerning the organisation of the collagen. Nevertheless, cellularity and vascularity could easily be analysed, and these features of tendinosis were therefore chosen as objective measurements in accordance with the already established protocol for evaluating this model regarding development of tendinosis-like tissue changes. ${ }^{17}$

As for the paratendinous tissue, an invasion of inflammatory cells was observed in the tissue from the exercised groups, especially in proximity to the myotendinous junction. Particularly, a marked cellularity of the paratenon was noted in the SP-injected group. In this group, a pronounced swelling of the paratendinous tissue, as seen macroscopically, was also observed. Further notes on inflammation are described below. Inflammatory cells were absent in the tendon tissue proper, as is typically the case in human tendinosis. ${ }^{16}$

\section{NK-1R immunoreactions and mRNA}

NK-1R (Figure 1A) and NK-1R-mRNA (Figure 1B) were seen in cells of blood vessel walls of the tendon tissue in the SP- and $\mathrm{NaCl}$-injected groups, as evidenced by immunohistochemistry and ISH, respectively. The reactions were mainly seen in the endothelium, but the smooth muscle cells also expressed the receptor. The negative control stainings showed no reactions (preabsorptions and sense staining, respectively). However, some vessels were immunonegative for NK-1R in all groups
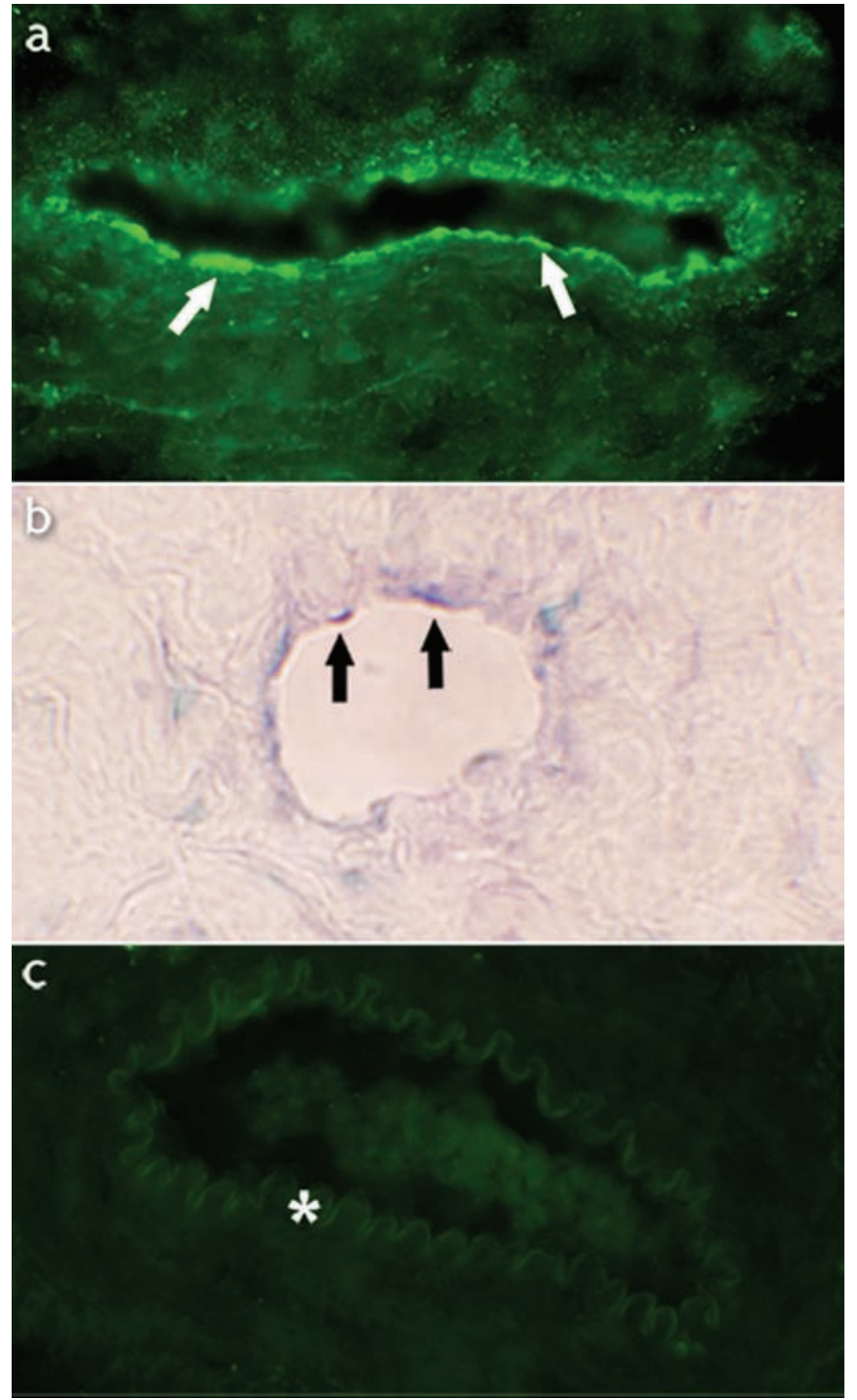

Figure 1 Presence of NK-1R and NK-1R mRNA in vessels of the rabbit Achilles tendon. (A) Transverse section of rabbit Achilles tendon tissue from the exercised leg of a rabbit in the substance $\mathrm{P}(\mathrm{SP})$-injected group stained for NK-1R. Reactions are seen in the endothelial layer of a large vessel (arrows). (B) Section from a rabbit in the SP-injected group (exercised leg) processed with in situ hybridisation, using digoxigenin-alkaline phosphatase detection, detecting NK-1R (TACR1) mRNA. Antisense staining. Fine endothelial reactions are seen in a blood vessel (arrows). (C) Tissue from a rabbit in the SP-injected group (exercised leg) processed for NK-1R immunohistochemistry. This vessel shows no NK-1R immunoreactivity in endothelial cells (asterisk). This shows that there was a diversity between different blood vessels concerning NK-1R reaction patterns (cf. A). A $63 \times / 0.95$ objective-equipped microscope was used for the immunofluorescence stainings and in situ hybridisation.

(Figure 1C). Paratendinous nerve fascicles, as well as inflammatory cells, showed NK-1R reactions (not shown). NK-1R immunoreactivity (Figure 2, inset), as well as NK-1R mRNA (Figure 2), were seen in the tenocytes in both injected groups.

For the untrained controls and 1-week group, it has previously been shown that these reactions are noticeable in the tendon tissue. ${ }^{22}$

\section{Quantification of tenocytes}

There was a significant increase in the number of tenocytes of the tendon tissue proper in the SP-injected group compared 


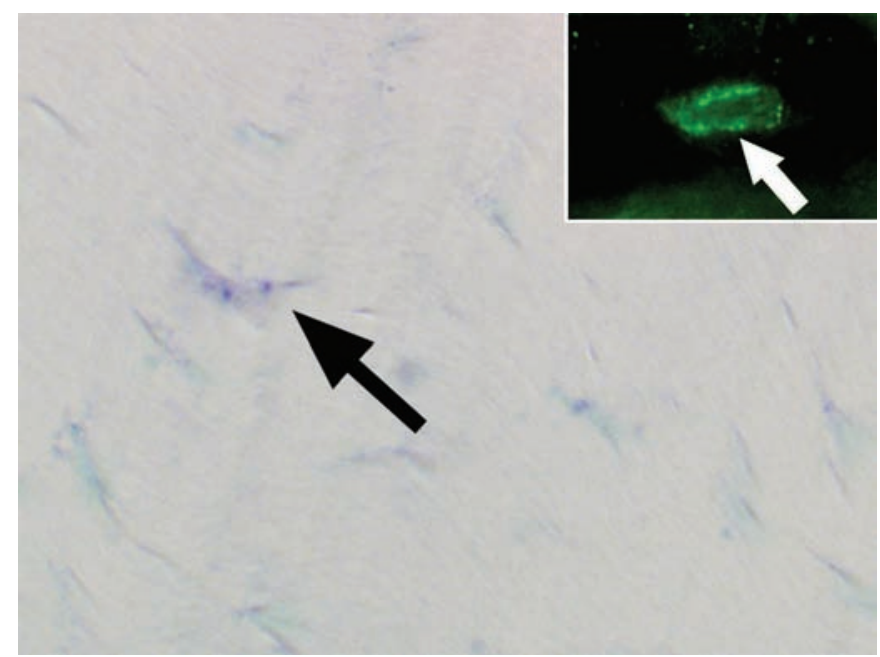

Figure 2 Section of Achilles tendon tissue from an animal in the substance P-injected group (exercised leg) processed with in situ hybridisation detecting NK-1R (TACR1) mRNA. Reactions are seen in tenocytes (arrow) of the tendon tissue proper when using an antisense probe cocktail and an alkaline phosphatase-labelled antibody detecting the probe cocktail (cf. Material and Methods). Inset: A tenocyte immunohistochemically stained for NK-1R displaying reactions in the cellular membrane. Achilles tendon tissue from a rabbit in the 1-week control group (exercised leg). A $63 \times / 0.95$ microscope objective was used.

with both the untrained controls and the 1 -week controls (Mann-Whitney U pairwise test; $p<0.05$, Figure 3). The $\mathrm{NaCl}$-injected group also showed increased tenocyte number compared with untrained controls. There was, however, no significant difference between the SP-injected group and the $\mathrm{NaCl}$ controls, or between the latter and the 1-week controls (Figure 3).

The median number of tenocytes quantified in the areas of biopsy $\left(283 \times 213 \mu \mathrm{m}^{2}\right)$ was 99 for the untrained controls (with the middle $50 \%$ of the observations, that is, the IOR, lying between 93 ( $\mathrm{O} 1=$ lowest quartile) and 101 (O3=highest quartile)) and 87 for 1-week controls (IOR: 78-101), see ref ${ }^{17}$. The numbers were 157 for the NaCl-injected group (IOR: 135173) and 160 for the SP-injected group (IOR: 144-175).

In the SP-injected group, comparisons were made between the tendons of both legs, both of which had been subjected to $\mathrm{SP}$ injections. No significant difference in the number of tenocytes was seen when comparing the exercised (right) with the non-exercised (left) legs (Figure 4). The non-exercised leg of the animals in the SP-injected group had a median number of tenocytes per quantified area of 155 (IOR: 153-164).

\section{Vascularity}

An increased vascularity was noted in the SP-injected group compared with untrained controls $(p=0.020$, Figure 5$)$. The median vascular score for the SP-injected group was 7.25 (IOR: 5.25-8.25) and for the untrained control group it was 3.50 (IOR: 3.00-4.00). There was no significant increase of the vascularity in the $\mathrm{NaCl}$ group (median: 4.00, IOR: 2.00-6.00) compared with the untrained controls, and no statistically significant difference in vascularity could be seen in any other analysis made between the groups (see Figure 5).

There was no significant difference between the median vascular grade of the tendons in the exercised leg and the grade of those in the unexercised leg in the SP-injected group.

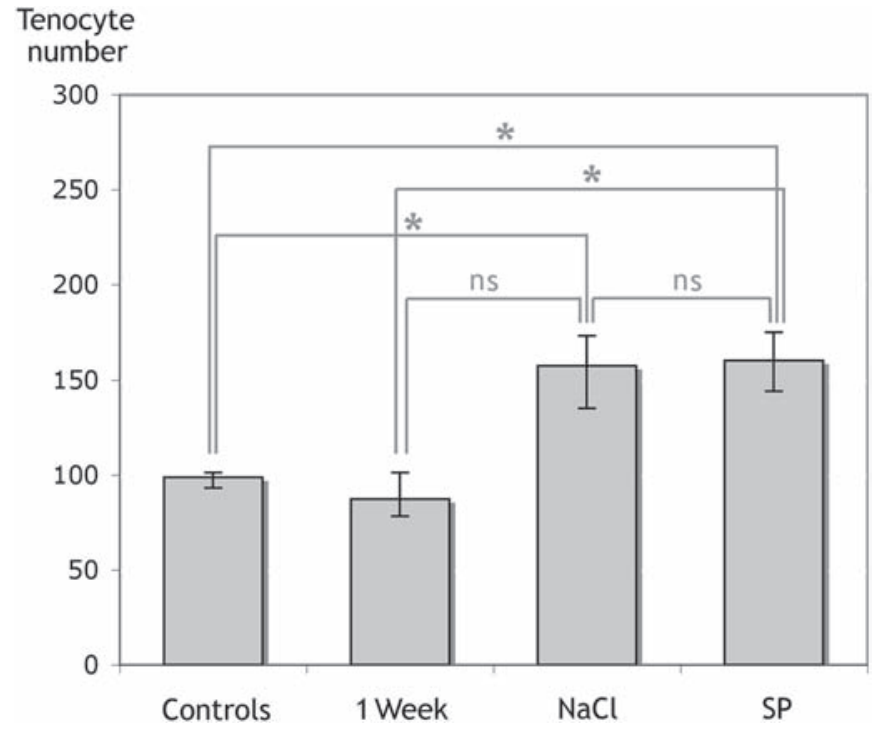

Figure 3 Tenocyte count of the right (exercised) leg of the four groups (untrained controls ('controls'), 1-week controls, $\mathrm{NaCl}$ injected and substance $\mathrm{P}(\mathrm{SP})$-injected groups). The median number of tenocytes in the counted area (size $283 \times 213 \mu \mathrm{m}^{2}$; mean of the counted number in three micrographs per tendon part) was significantly $(* p<0.05)$ increased in the injected groups compared with the untrained control and/or 1-week groups. No significant difference (ns) was found between the $\mathrm{NaCl}$ - and SP-injected groups. Error bars indicate IQR. Mann-Whitney U test was used.

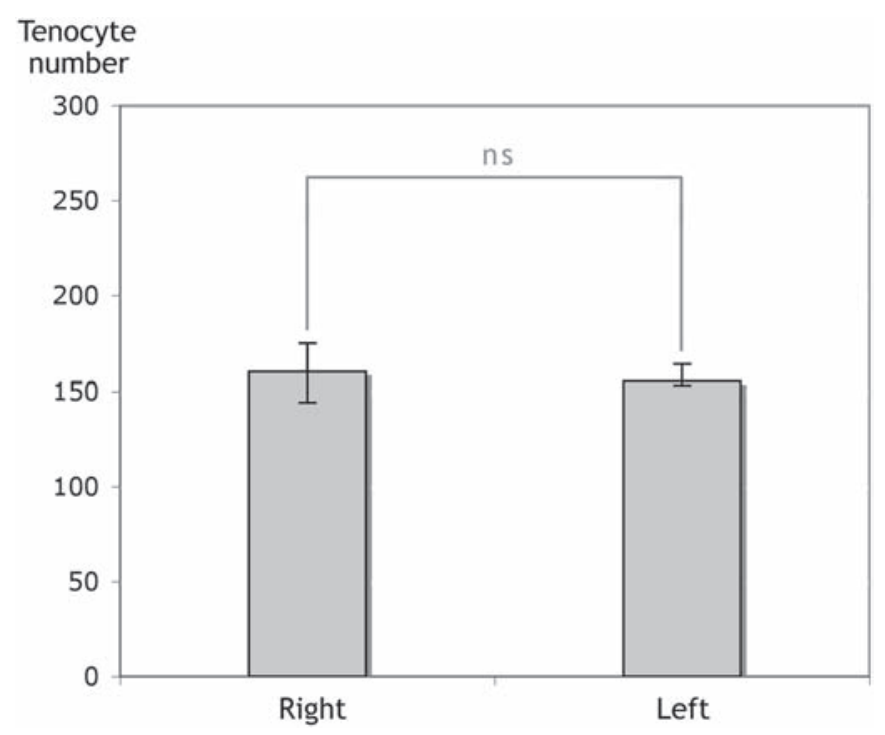

Figure 4 Tenocyte count of the right and left legs of the animals in the substance P-injected group. No significant difference (ns) in the median number of tenocytes could be seen between the exercised (right) and non-exercised (left) legs of the same rabbits. Wilcoxon signed rank test. Error bars indicate IQR.

\section{Paratendinous inflammation}

Paratendinous inflammation was noted for all exercised groups. The infiltration of inflammatory cells in the paratendinous tissue was more extensive in the SP-injected group compared with the $\mathrm{NaCl}$ controls, although there were also inflammatory cells in the paratendinous tissue of the latter group as well as in that of the 1-week controls.

The most apparent infiltration was seen in the loose connective tissue at the level of the myotendinous junction. When 


\section{Grade}

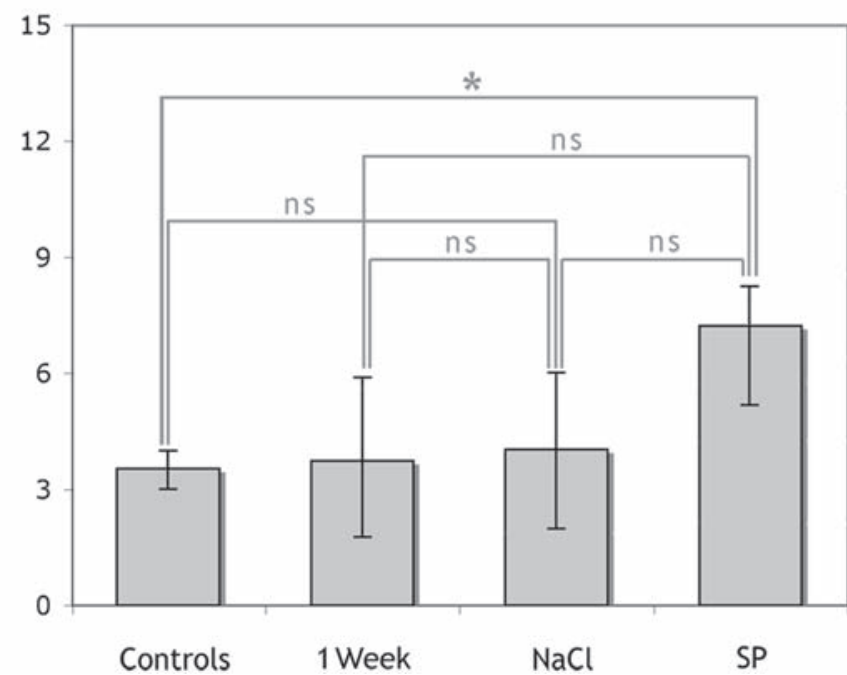

Figure 5 Vascularity of the tendon tissue. Semiquantitative grading of vascular structures using the modified Bonar scale. The median grades for vascularity of the combined score for the three parts of the tendon (mean grade for both tendons when available) showed a significant $\left({ }^{*} p<0.05\right)$ difference only between the untrained control group ('controls') and the substance $\mathrm{P}(\mathrm{SP})$-injected group. MannWhitney $U$ test. Error bars indicate IQR.

using blinded grading of the level of inflammation (see Material and Methods), and Mann-Whitney U statistical test, to compare the two injected groups, a difference concerning grade of inflammation was seen for the myotendinous junction, in which the grade of inflammation was significantly higher for the SP-injected group compared with the $\mathrm{NaCl}$ control group (Figure 6; SP group: median $=2.75$, IOR=2.38-3.00, $\mathrm{NaCl}$ control group: median $=1.50$, IOR $=0.50-1.75$ ). No significant difference could be seen between the groups when looking at the distal parts of the tendon or the middle portion.

\section{DISCUSSION}

In this study, we show that paratendinous SP injections accelerate the development of the tendinosis-like changes studied (tenocyte hypercellularity and vascular proliferation) in the Achilles tendon in response to an overuse protocol during a time period of 1 week. We have previously shown that these changes do not develop until after a minimum of 3 weeks of exercise in this model, if no injections are given. ${ }^{17}$ The observations presented here thus support an involvement of SP in the processes that occur in the early development of tendinosis, and are in line with previous studies showing that SP contributes towards tendon repair. ${ }^{3}$ Through stainings for the preferred receptor for SP, the tachykinin receptor NK-1R, an anatomic basis was provided for widespread SP effects in the injected rabbit tendon tissue, including effects on blood vessels, nerve structures, inflammatory cells and tenocytes. This is in accordance with previous findings in human Achilles tendon tissue, ${ }^{9}{ }^{10}$ further underlining that this rabbit model is valid for studies on SP effects in tendon diseases.

Surprisingly, a significant increase in tenocyte proliferation was noted not only in the SP-injected group but also in the group injected with $\mathrm{NaCl}$ (saline). There was, however, no increase in vascularity in the latter group. $\mathrm{NaCl}$ is considered a suitable control when analysing effects of administrated substances. However, in this study, $\mathrm{NaCl}$ injections promoted
Grade

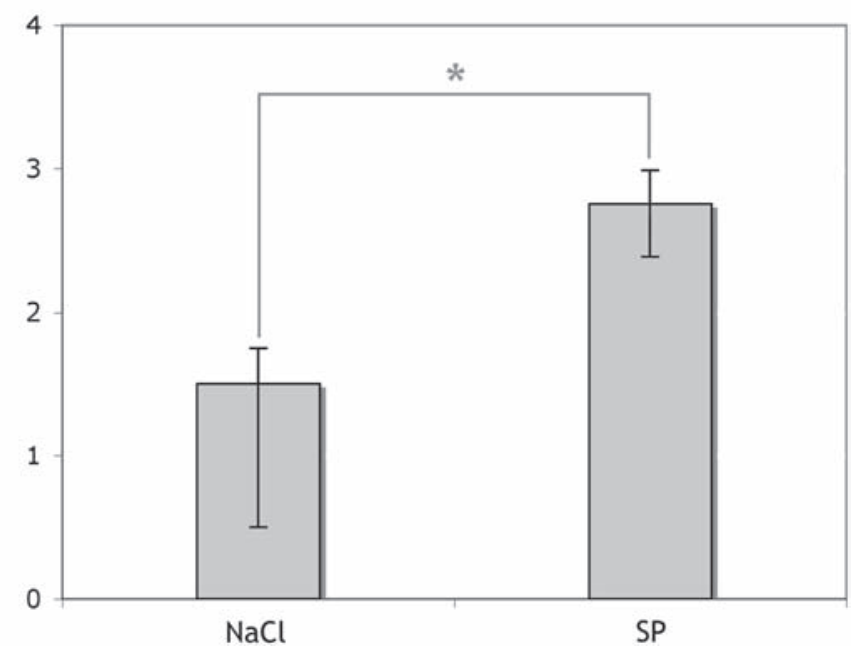

Figure 6 Degree of inflammation in the paratenon of the proximal part of the Achilles tendon (close to the myotendinous junction). A significant difference $\left({ }^{*} p<0.05\right)$ in the semiquantitative grading of inflammatory infiltrates could be seen, with a higher median grade for the substance $\mathrm{P}(\mathrm{SP})$-injected group, when compared with the $\mathrm{NaCl}$ injected group. Mann-Whitney U test. Error bars indicate IQR.

hypercellularity almost to the same degree as did SP. One explanation for this finding is that the injections increased local tissue pressure triggering an adaptive response, that is, cellular proliferation. The administered volume of $1 \mathrm{ml}$ (SP or $\mathrm{NaCl}$ ) in the connective tissue around the tendon might exert a notable increase in intratendinous pressure. Alternately, the proliferation of tenocytes may have been secondary to a possible increase in local release of SP or inflammatory cytokines caused by the $\mathrm{NaCl}$ injections themselves; a phenomenon previously shown to occur in a mouse model in which intradermal saline injections in the ear elevated tissue SP levels. ${ }^{23}$ Moreover, it has been theorised that an important clinical aspect of injection treatments for tendinosis may be due to a volume effect. ${ }^{24}$ ${ }^{25}$ However, the mechanism of this volume effect is currently unknown.

The observation that the vascularity of the tendon was increased in the SP-injected group was not an unexpected result, given the well-known angiogenic effects of SP. However, if $\mathrm{NaCl}$ injections induced tenocyte proliferation by stimulating endogenous SP production, as speculated in the previous section, then why did such a possible increased $\mathrm{SP}$ production due to $\mathrm{NaCl}$ injections not also affect vascular proliferation? An explanation may be that the angiogenic effect was mostly related to effects of SP in the paratendinous region, including effects on vessel ingrowth into tendon tissue and proinflammatory effects. Indeed, this study shows that SP injections led to significantly higher degrees of paratendinous inflammatory infiltration than $\mathrm{NaCl}$ injections. Thus, it is possible that the more pronounced vascular proliferation in the SP-injected group compared with $\mathrm{NaCl}$ controls could potentially be ascribed to direct angiogenic effects of $\mathrm{SP}, 826$ in parallel with the known proinflammatory effect of SP, including mast cell activation. In fact it has been proposed that tendinosis might occur secondarily to inflammation of the paratendinous structures. ${ }^{27}$

Interestingly, SP injections, being given in both legs, increased the tenocyte number and vascular density not only in the exercised leg, but also in the unexercised leg (see 


\section{What is already known on this topic}

- The tissue changes seen in tendinosis include cellular proliferation and angiogenesis.

- Patients with tendinopathy show signs of an upregulated substance $P(S P)$ system within the diseased tendon.

- SP has established angiogenic and cell proliferative effects.

\section{What this study adds}

- Exogenously administered SP accelerates tenocyte proliferation and angiogenesis in the Achilles tendon, and causes a paratendinous inflammation, during tendinosis development in a rabbit model.

- SP is likely to be involved in the development of tendinosis, and is thus a possible target for intervention treatments.

Figure 4). In our previous study of this re-established animal model, ${ }^{17}$ we showed that the unexercised leg was not a suitable control, as there were noticeable bilateral tendinosis-like changes in response to unilateral training, possibly due to central neuronal mechanisms; albeit that also on the resting side these changes were seen only after a minimum of 3 weeks of exercise if no injections were given. The present study demonstrates that SP injections lead to tendinosis-like changes in the rabbit tendon also without the tendon being directly exercised. Consequently, SP alone, or possibly in combination with transferred effects of exercise in the contralateral leg, seems to produce the tissue changes, although this is not controlled for in the present study.

Gender aspects are of importance in tendon research. Early in vitro studies on rabbit tendon tissue cells showed that mRNA levels of different important tissue regulators, such as insulinlike growth factor-2, tissue inhibitor of metalloproteinase-1 and urokinase, are influenced by exogenously administered SP, and, most interestingly, that the changes seen are gender specific. ${ }^{28}$ When assessing the outcome of the present study, it must therefore be stressed that only female animals were used throughout the studies.

Summarising the results from this study, we found that injections of SP in combination with only 1 week of tendon overuse caused histopathological changes similar to those found in human Achilles tendinosis (angiogenesis and hypercellularity). We also observed a pronounced paratendinous inflammatory response to the injections. Saline injections had a statistically significant effect on the number of tenocytes in the tendon tissue proper, but caused no increase in vascularity or paratendinous inflammation compared with the non-injection group. It is obvious that SP may participate in the events that occur in tendinosis development, which is clinically interesting since substances are available for blocking of SP effects via NK-1R.

Acknowledgements The authors thank Ms Ulla Hedlund, Mr Adrian Lamouroux and Ms Fellon Robson-Long for excellent technical service. The authors also thank Dr James Edmund Gaida, Prof Håkan Alfredson and Dr Clas Backman for co-operation on the animal model.

Funding Financial support has been obtained from the Faculty of Medicine at Umeå University (SF, PD), the Sigurd and Elsa Golje Memorial Foundation (PD), the Tore Nilsson Foundation for Medical Research (PD), the J C Kempe and Seth M Kempe Memorial Foundations, Örnsköldsvik (SF) and Worksafe, British Columbia, Canada (AS).

\section{Competing interests None.}

Provenance and peer review Not commissioned; externally peer reviewed.

\section{REFERENCES}

1. Scott A, Bahr R. Neuropeptides in tendinopathy. Front Biosci 2009;14:2203-11.

2. Bring DK, Reno C, Renstrom P, et al. Joint immobilization reduces the expression of sensory neuropeptide receptors and impairs healing after tendon rupture in a rat model. J Orthop Res 2009;27:274-80.

3. Burssens $\mathbf{P}$, Steyaert A, Forsyth $\mathrm{R}$, et al. Exogenously administered substance $P$ and neutral endopeptidase inhibitors stimulate fibroblast proliferation, angiogenesis and collagen organization during Achilles tendon healing. Foot Ankle Int 2005;26:832-9

4. Carlsson 0, Schizas N, Li J, et al. Substance P injections enhance tissue proliferation and regulate sensory nerve ingrowth in rat tendon repair. Scand J Med Sci Sports

5. Steyaert AE, Burssens PJ, Vercruysse CW, et al. The effects of substance P on the biomechanic properties of ruptured rat Achilles' tendon. Arch Phys Med Rehabil 2006;87:254-8

6. Harrison S, Geppetti P. Substance P. Int J Biochem Cell Biol 2001;33:555-76.

7. Lam FF, Yip AL. Unique gradual and sustained vasodilator response to substance $P$ in the rabbit knee joint. Eur J Pharmacol 2000;400:327-35.

8. Fan TP, Hu DE, Guard S, et al. Stimulation of angiogenesis by substance P and interleukin-1 in the rat and its inhibition by NK1 or interleukin-1 receptor antagonists. Br J Pharmacol 1993;110:43-9.

9. Andersson G, Danielson $\mathrm{P}$, Alfredson $\mathrm{H}$, et al. Presence of substance $\mathrm{P}$ and the neurokinin-1 receptor in tenocytes of the human Achilles tendon. Regul Pept 2008:150:81-7.

10. Forsgren S, Danielson P, Alfredson H. Vascular NK-1 receptor occurrence in normal and chronic painful Achilles and patellar tendons: studies on chemically unfixed as well as fixed specimens. Regul Pept 2005;126:173-81.

11. Schubert TE, Weidler $C$, Lerch $K$, et al. Achilles tendinosis is associated with sprouting of substance P positive nerve fibres. Ann Rheum Dis 2005;64:1083-6.

12. Scott A, Lian $\emptyset$, Bahr R, et al. Increased mast cell numbers in human patellar tendinosis: correlation with symptom duration and vascular hyperplasia. $\mathrm{Br} \mathrm{J}$ Sports Med 2008;42:753-7.

13. Ackermann PW, Li J, Lundeberg T, et al. Neuronal plasticity in relation to nociception and healing of rat achilles tendon. J Orthop Res 2003;21:432-41.

14. Peers KH, Lysens RJ. Patellar tendinopathy in athletes: current diagnostic and therapeutic recommendations. Sports Med 2005;35:71-87.

15. Riley G. The pathogenesis of tendinopathy. A molecular perspective. Rheumatology (Oxford) 2004;43:131-42.

16. Khan KM, Cook JL, Bonar F, et al. Histopathology of common tendinopathies. Update and implications for clinical management. Sports Med 1999;27:393-408.

17. Andersson G, Forsgren S, Scott A, et al. Tenocyte hypercellularity and vascular proliferation in a rabbit model of tendinopathy: contralateral effects suggest the involvement of central neuronal mechanisms. Br J Sports Med 2011;45:399-406.

18. Backman C, Boquist L, Fridén J, et al. Chronic achilles paratenonitis with tendinosis: an experimental model in the rabbit. J Orthop Res 1990;8:541-7.

19. Backman C, Fridén J, Widmark A. Blood flow in chronic Achilles tendinosis. Radioactive microsphere study in rabbits. Acta Orthop Scand 1991;62:386-7.

20. Cook JL, Feller JA, Bonar SF, et al. Abnormal tenocyte morphology is more prevalent than collagen disruption in asymptomatic athletes' patellar tendons. J Orthop Res 2004;22:334-8.

21. Panoskaltsis-Mortari A, Bucy RP. In situ hybridization with digoxigenin-labeled RNA probes: facts and artifacts. BioTechniques 1995;18:300-7.

22. Backman LJ, Andersson G, Wennstig G, et al. Endogenous substance $P$ production in the Achilles tendon increases with loading in an in vivo model of tendinopathy: peptidergic elevation preceding tendinosis-like tissue changes. J Musculoskelet Neuronal Interact 2011:11:133-40.

23. Chen SC, Tsai MH, Wang HJ, et al. Involvement of substance $P$ and neurogenic inflammation in arsenic-induced early vascular dysfunction. Toxicol Sci 2007:95:82-8.

24. Humphrey J, Chan 0, Crisp T, et al. The short-term effects of high volume image guided injections in resistant non-insertional Achilles tendinopathy. J Sci Med Sport 2010;13:295-8.

25. Zeisig E, Fahlström M, Ohberg L, et al. Pain relief after intratendinous injections in patients with tennis elbow: results of a randomised study. Br J Sports Med 2008:42:267-71.

26. Ziche M, Morbidelli L, Masini E, et al. Nitric oxide mediates angiogenesis in vivo and endothelial cell growth and migration in vitro promoted by substance P. J Clin Invest 1994;94:2036-44.

27. Bring DK, Heidgren ML, Kreicbergs A, et al. Increase in sensory neuropeptides surrounding the Achilles tendon in rats with adjuvant arthritis. J Orthop Res 2005;23:294-301.

28. Hart DA, Kydd A, Reno C. Gender and pregnancy affect neuropeptide responses of the rabbit Achilles tendon. Clin Orthop Relat Res 1999;365:237-46. 